\title{
Testicular schistosomiasis as differential diagnosis for testicular nodules: a case report
}

\author{
Tiago Neves Ferreira ${ }^{[1]}$, Hana Caroline Morais Higa ${ }^{[2]}$, Ronaldo Garcia Rondina ${ }^{[1]}$, \\ Márcio Maia Lamy Miranda ${ }^{[3]}$, José Nivaldo Fin ${ }^{[3]}$, Marcos Lyra Kaddoun ${ }^{[1]}$, \\ Maria Carmen Ferreira Lopes Silva Santos ${ }^{[4]}$ and Rodrigo Stênio Moll de Souza ${ }^{[5]}$
}

[1]. Hospital Universitário Cassiano Antônio Moraes, Universidade Federal do Espírito Santo, Vitória, Espírito Santo, Brasil. [2]. Curso de Medicina, Universidade Federal do Espírito Santo, Vitória, Espírito Santo, Brasil. [3]. Serviço de Urologia, Hospital Universitário Cassiano Antônio Moraes, Universidade Federal do Espírito Santo, Vitória, Espírito Santo, Brasil. [4]. Departamento de Patologia, Universidade Federal do Espírito Santo, Vitória, Espírito Santo, Brasil. [5]. Serviço de Radiologia, Hospital Universitário Cassiano Antônio Moraes, Universidade Federal do Espírito Santo, Vitória, Espírito Santo, Brasil.

\begin{abstract}
Despite its infrequent occurrence, testicular schistosomiasis forming pseudo-tumors can be considered in the differential diagnosis of testicular tumors, especially in areas where the parasitic disease is endemic. In this report, we present a case of testicular schistosomiasis caused by Schistosoma mansoni and mimicking a testicular neoplasm. We describe the patterns of a testicular nodule on ultrasonography and magnetic resonance images in a 46-year-old man. The nodule was removed after a pre-operative diagnosis of a non-malignant lesion. Histology demonstrated granulomas with epithelioid macrophages and eosinophils around S. mansoni eggs within a fibrous tissue that formed a nodular structure.
\end{abstract}

Keywords: Schistosomiasis. Testis. Testicular schistosomiasis. Tumor. Schistosoma mansoni. Radiology.

\section{INTRODUCTION}

Testicular nodes have several potential origins, with neoplastic causes the most important diagnoses. However, when confronted with a testicular mass in a region of endemic schistosomiasis, this parasitic disease might be considered because it can cause a local granulomatous inflammation that presents as a mass similar to a testicular neoplasm.

In this article, we report a case of testicular schistosomiasis with emphasis on lesion patterns on ultrasonography (US) and magnetic resonance imaging (MRI).

\section{CASE REPORT}

A 46-year-old male patient, originally from Itororó, State of Bahia, attended the urology service at the University Hospital Cassiano Antônio Moraes from the Federal University of Espírito Santo, Brazil, with the initial complaint of difficulty urinating with progressive worsening, mainly during the previous year. He had no other complaints.

\footnotetext{
Corresponding author: Dr. Tiago Neves Ferreira. Avenida Marechal Campos 1355, Bairro Santos Dumont, 29040-091 Vitória, Espírito Santo, Brasil. Phone: 5527 3335-7222; 5527 3335-7000

e-mail:nftiago@gmail.com; hanahiga@gmail.com

Received 27 October 2014

Accepted 11 May 2015
}

During the physical exam, the glans could not be exposed due to phimosis. The testicles were topical. A node of $1 \mathrm{~cm}$ diameter was detected with hardened consistency in the right testicle, with no other changes.

The leukocyte count was 4,270 cells $/ \mathrm{mm}^{3}$ and indicated important eosinophilia (491 cells $/ \mathrm{mm}^{3} ; 11.5 \%$ ); however, the parasitological feces examination was negative for protozoa and helminthes. The tumor markers human chorionic gonadotropin and alpha-fetoprotein were negative, and lactate dehydrogenase was slightly high (288, VR: 220).

Ultrasound (US) with Doppler examination of the scrotum showed a hypoechoic nodule image with regular contours and peripheral and central vascularity measuring $1.4 \times 1.0 \times 1.0 \mathrm{~cm}$ in the lower third of the right testicle (Figure 1).

On MRI of the testicles, a solid and hypervascularized nodule of indeterminate nature and bilateral ectasia of the veins of the pampiniform plexus were observed on the right testicle (Figure 2).

The patient was hospitalized and, due to a spare characteristic neoplasm on imaging, underwent a right partial orchiectomy with perioperative freezing; the result was negative for malignancy. We performed a circumcision and cystostomy after unsuccessfully passing a urethral catheter. There was almost nonexistent exposure of the glans, with plenty of grip during retraction of the foreskin, making it difficult to pass the catheter. The excisional biopsy of the right testicular nodule was sent for pathological examination, and the final diagnosis was chronic granulomatous inflammation with eosinophilia around Schistosoma mansoni eggs. 


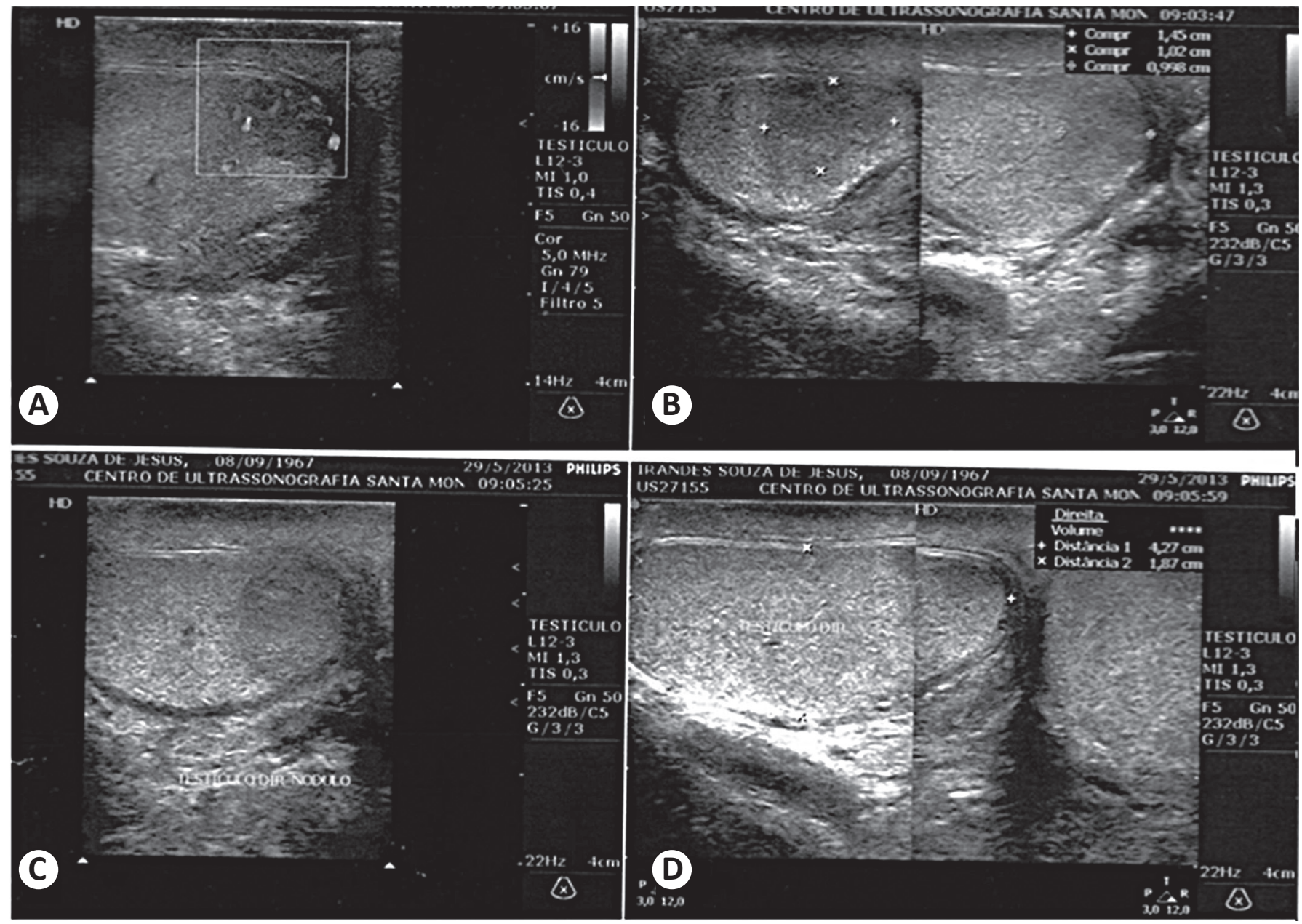

FIGURE 1 - Ultrasonography of the right testis: A: hypoechoic nodule in the lower pole, with predominantly peripheral flow on color Doppler. B: The nodule measures $1.5 \times 1.0 \times 0.9 \mathrm{~cm}$ with a partially defined and irregular contour. C: The nodule has a heterogeneous echo texture. D: The diameters of the right testicle $\mathrm{C}$ remain preserved.



FIGURE 2 - Magnetic resonance imaging: A: T2 sequence of a peripheral nodule in the right testis with hypointense, irregular outlines and well-defined limits, without promoting an expansive effect. B: T1 sequence of fat saturation without paramagnetic contrast in a peripheral nodule with mild hyperintensity. $C$ : T1 sequence of fat saturation with paramagnetic contrast of a lateral peripheral nodule is observed in the right testicle showing intense enhancement; discrete peripheral enhancement in the tunica albuginea is also observed. 


\section{DIscussion}

Although schistosomiasis is endemic in many countries, testicular involvement is extremely rare. In an autopsy study in an endemic area in Brazil, testicular involvement was found in $3.2 \%$ of the schistosomiasis cases, indicating that these lesions are commonly subclinical ${ }^{(1)}$. Testicular involvement is attributed to the migration of eggs through venous channels between the internal spermatic and mesenteric veins ${ }^{(2)}$, inducing an immune response with granuloma formation and associated fibrotic changes ${ }^{(3)(4)}$. This lesion can mimic a malignant lesion presenting with painless small solid nodules.

Echographically, the schistosomal lesion appears as a well delimited solid testicular mass with a heterogeneous echotexture or hypoechogenicity and shows increased vascularity on color Doppler flow imaging, which is an appearance identical to that of most testicular malignancies ${ }^{(3)(5)(6)}$.

Magnetic resonance imaging might be better than US to show intratesticular abnormalities; however, neither technique allows differentiation between benign and malignant disorders ${ }^{(7)}$.

There are few studies describing MRI findings of testicular schistosomiasis (TS) in the literature. Lopes et al ${ }^{(8)}$ described, in a case report, an irregular tunica albuginea signal on the posterior side of the testis without evidence of a focal nodular lesion ${ }^{(8)}$. Hassan et al reported an extratesticular mass of low signal intensity on T2-weighted imaging, indicating a fibrous tissue component. No intratesticular masses of abnormal signal intensity or enhancement were present ${ }^{(2)}$. In our case, we report a solid and hypervascularized intratesticular nodule associated with bilateral ectasia of the veins of the pampiniform plexus.

The recognition of TS as a differential diagnosis for testicular tumors is crucial to avoid unnecessary orchiectomies ${ }^{(9)}$. The authors believe that the indication for radical orchiectomy should be carefully reviewed to avoid unnecessary invasive surgeries, especially in patients of fertile age and in schistosomiasis endemic areas. In this context, a partial orchiectomy from the inguinal approach with perioperative freezing can be a safe and effective approach for both diagnosis and treatment of benign lesions; it also allows conversion to a radical orchiectomy for neoplasms diagnosed during freezing.

\section{REFERENCES}

1. Gonçalves EC, Fonseca AP, Pitella JE. Frequency of schistosomiasis mansoni, of its clinicopathological forms and of the ectopic locations of the parasite in autopsies in Belo Horizonte, Brazil. J Trop Med Hyg 1995; 98:289-295.

2. Hassan A, El-Mogy S, Zalata K, Mostafa T. Testicular schistosomiasis: a case study. Fertil Steril 2011; 95:2124.

3. Shebel HM, Elsayes KM, Abou El, Atta HM, Elguindy YM, El-Diasty TA. Genitourinary schistosomiasis: life cycle and radiologic-pathologic findings. Radiographics 2012; 32:1031-1046.

4. Moudgil A, Kosut J. Urinary schistosomiasis: an uncommon cause of gross hematuria in the industrialized countries. Pediatr Nephrol 2007; 22:1225-1227.

5. Soans B, Abel C. Ultrasound appearance of schistosomiasis of the testis. Australas Radiol 1999; 43:385-387.

6. Woodward PJ, Schwab CM, Sesterhenn IA. Extratesticular scrotal masses: radiologic-pathologic correlation. Radio Graphics 2003; 23:215-240.

7. Thurnher S, Hricak H, Carroll PR, Pobiel RS, Filly RA. Imaging of the testis: comparison between MR imaging and US. Radiology 1998; 167:631-636.

8. Lopes RI, Leite KR, Prando D, Lopes RN. Testicular schistosomiasis caused by Schistosoma mansoni: a case report from Brazil. Braz J Infect Dis 2007; 11:523-524.

9. Periyasamy P, Subramaniam SR, Rajalingham S. An increasingly notorious mimicker of testicular tumours; crossing borders. BMJ Case Rep 2011; pii: bcr0920114816. doi: 10.1136/bcr.09.2011.4816. 